\title{
Human-Factor Risk Mitigation in Outdoor Climbing Areas: Survey of Existing Policies in Regulated Climbing Areas
}

\author{
Jourdan H. Meltzer, BA ${ }^{1}$; Joseph D. Forrester, MD, $\mathrm{MSc}^{2}$ \\ ${ }^{1}$ Warren Alpert School of Medicine, Brown University, Providence, Rhode Island; ${ }^{2}$ Department of Surgery, Stanford University, Stanford, \\ California
}

Introduction-Popular climbing areas in the United States are frequently on public lands under administrative purview of land management entities. During climbing, climbers may be subject to injury. This study aimed to describe existing climbing risk mitigation tactics used to protect climbers by oversight agencies responsible for these public climbing areas and to identify strategies perceived by the oversight agency to be successful.

Methods-We identified publicly managed US bouldering, sport, or traditional climbing areas through a state-by-state search of Mountain Project. We assessed climbing-related visitation, injury, rescue, and risk mitigation strategies using a 10-item survey targeting land-use managers, rangers, outdoor recreation planners, and park managers. Quantitative analysis included univariate and multivariate analysis. Qualitative analyses of survey responses with thematic grouping of mitigation interventions were performed.

Results-One hundred fifty-seven publicly managed US climbing areas were contacted, and 76 $(48 \%)$ provided data. Thirty-six $(47 \%)$ of those that provided data stated that programs are in place to reduce climbing-related injury. There were no associations between demographic variables and the presence of risk mitigation strategies. Four themes of climbing risk mitigation strategies emerged: coordination with climbing coalitions ( $25 \%$ of respondents), permitting ( $22 \%$ of respondents), publication of accident reports (22\% of respondents), and preventative search and rescue (17\% of respondents).

Conclusions-Nearly half of survey respondents reported having climbing risk mitigation programs. There is opportunity to assess the efficacy of risk mitigation strategies through intra-area and inter-area assessments. A centralized climbing injury database may prove useful for assessing the efficacy of and need for risk mitigation techniques.

Keywords: preventative search and rescue (PSAR), injury prevention, National Parks, public lands

\section{Introduction}

Rock climbing, mountaineering, and indoor rock climbing are increasingly popular recreational activities in the United States, with over 6 million participants annually. ${ }^{1-3}$ Climbing is increasingly accessible to the general population, and participants are transitioning from climbing indoors to climbing outdoors. ${ }^{1}$ A range of injuries can occur while climbing, from falls to stressrelated musculoskeletal injuries. ${ }^{1,4}$ Climbers may also be

Corresponding author: Joseph D. Forrester, MD, MSc, 300 Pasteur Drive, H3591, Stanford, CA 94305; e-mail: jdf1@stanford.edu.

Submitted for publication December 2020.

Accepted for publication August 2021. at risk for exposure-based conditions, including hypothermia, sunburn, and dehydration, depending on conditions and climber preparedness.

Although climbing can be done anywhere rocks are present, popular climbing areas in the United States frequently fall under the administrative purview of the National Park Service (NPS), Bureau of Land Management (BLM), US Department of Agriculture Forest Service (USDAFS), state parks, or city or county parks. There are limited published data describing climbing risk mitigation tactics used by these oversight entities to reduce rates of climbing-related injury and rescue. We sought to describe existing risk mitigation tactics used by oversight agencies responsible for these public climbing 
areas, with the goal of identifying strategies perceived to be successful.

\section{Methods}

Research protocols were approved by Stanford University and determined to be exempt after institutional review board review. We identified climbing areas for study via a systematic state-by-state search of the Mountain Project. The Mountain Project is a widely used, regularly updated website serving as a free online guidebook for over 170,000 climbing routes worldwide, relying on regional administrators and real-time usergenerated beta, photos, topographic maps, and comments. Navigating through the home page for each state, we captured publicly managed (under the purview of the NPS, BLM, USDAFS, and local city, county, and state park services) climbing areas. With further review of climbing areas, we excluded areas that primarily contained mountaineering or ice climbing routes, aiming for a sample of areas with a predominance of bouldering, sport, or traditional climbing.

Through area web pages (eg, general NPS, BLM, USDAFS, and local city, county, and state park services) and outreach to area ranger stations and offices, we collected contact information for individuals and/or offices with knowledge of or responsibility for climbingspecific risk and objectives in the area. We made $\geq 3$ separate attempts-over at least 2 forms of communication (phone and email) - to contact the individual or office of interest at each area. Once contact was made, we offered interviewees the option of anonymity and the choice to complete our survey either online or by phone.

Our 10-item survey was designed to assess climbingrelated visitation, injury, rescue, and risk mitigation strategies. Quantitative data obtained included climbingspecific visitor volumes, frequency of climbing-related injuries, and frequency of climbing-related rescues. Qualitative data collected included climbing-specific risks, tools used to minimize climbing-related injury, and area-specific concerns to provide context for risk mitigation tactics. Survey application and data collection ranged from April 1, 2020 to June 30, 2020.

For quantitative data analysis, we categorized continuous data into high, medium, and low. The visitation index was derived from stratifying reported annual climbing visitation from each area, using logarithmic numerical cutoffs: low ( $\leq 500$ climbing visitors), medium (500-7000 climbing visitors), and high (>7000 climbing visitors). Land area (in square kilometers) was defined as the complete area of a land management track obtained from official park reports or the GeoHack GIS aggregator. ${ }^{5-10}$ Cutoffs were created to reflect natural clustering of the data set: small $\left(<9.5 \mathrm{~km}^{2}\right)$, medium $\left(9.5-450 \mathrm{~km}^{2}\right)$, and large $\left(>450 \mathrm{~km}^{2}\right)$. Regions were defined by the 9 US Census Divisions. ${ }^{11}$ A composite risk index (reported as low, medium, or high) was generated by stratifying ratios of injuries and rescues to climbing visitors using logarithmic numerical cutoffs. Groups were as follows: low $(<10$ risk events [rescue and/or injury] per 10,000 climbing visitors), medium (10-14 risk events per 10,000 climbing visitors), and high (>14 risk events per 10,000 climbing visitors).

Data are presented as median with range. Univariate analysis was performed using the Fisher exact test. Multivariate logistic regression was used to assess predictors of the existence of risk mitigation plans using predetermined quantitative variables, including composite risk index, climbing region, visitation volume, and land area. The a priori $P$-value considered significant was $P \leq 0.05$. For qualitative data, we examined recurrent themes and identified choice quotes from survey respondents encapsulating approaches to risk management, obstacles faced, and tools to administer climbing risk mitigation programs and protocols. We used Stata 15.0 (StataCorp, College Station, TX) for analysis.

\section{Results}

We identified 159 climbing areas that met our inclusion criteria. Of these, 2 areas were removed from further evaluation because climbing was officially banned. We did not receive responses from 51 areas (32\%). One hundred six $(68 \%)$ responded, each representing a separate climbing area. Of those, $76(72 \%)$ provided data and $30(28 \%)$ refused to provide quantitative data in response to the survey. Thirty-four $(45 \%)$ of the 76 that provided data answered $100 \%$ of the survey questions. The most common job title of the survey respondent ( 20 of $76 ; 26 \%$ ) was ranger (including chief rangers, district rangers, lead and supervisory climbing rangers, backcountry rangers, and interpretive rangers). Areas of study were distributed across 9 US Census Regions (Figure 1).

Among 39 areas that reported risk-related data, there were 2.1 injuries per 10,000 climbing visitors (range: $0-50$ ) and 0.03 injuries per 10,000 annual visitors (range: $0-8.6)$. There were 1.1 rescues per 10,000 climbing visitors (range: $0-114$ ) and 0.02 rescues per 10,000 annual visitors (range: $0-0.2$ ). The median land mass of climbing areas of study was $64.8 \mathrm{~km}^{2}$ (range: 0.02-24,280). There were no associations between 


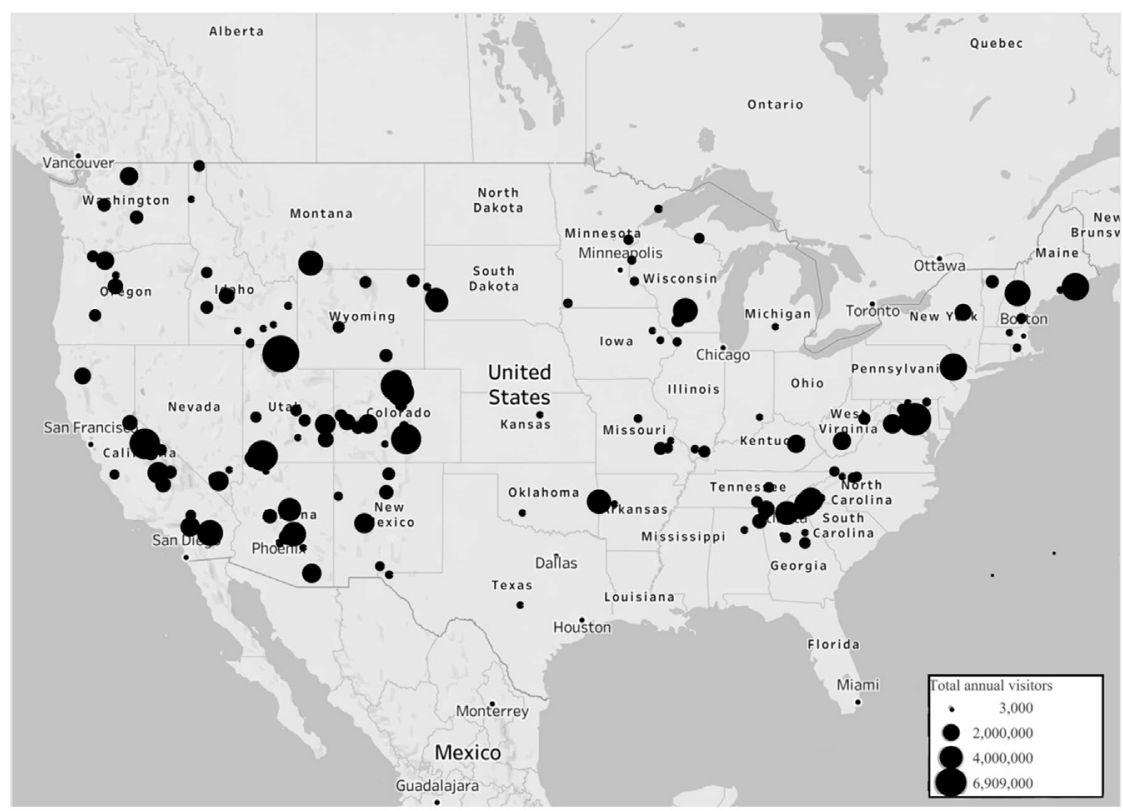

Figure 1. Map of 157 climbing areas of study. Map based on longitude and latitude. Total annual visitation (range: $3000-6,909,000)$ is scaled in the size of dots.

demographic variables and the presence of risk mitigation strategies (Table 1).

Respondents had heterogeneous responses regarding common geospatial locations of injury. Forty-one percent (31 of 76) stated they were unaware of common locations. Some reported this was based on a general lack of information about climbing-related injury and rescue in their area of governance. Others stated that an overall scarcity of climbing-related injury and rescue rendered them unable to identify any common location-based trends. Among respondents who reported trends in climbing-related injury, $13 \%$ (5 of 38 ) stated more difficult climbing-particularly overhung climbing of high grades $\left(5.11^{+}\right)$-contributed to climbing-related injuries and rescues. Two respondents reported increased difficulty of accessible climbing instead contributed to lower rates of injury and less need for rescue. Similarly, 4 respondents (11\%) stated that injuries were more common in areas with moderate grades (5.6-5.11) and easy climber access.

\section{RISK MITIGATION STRATEGIES: OVERVIEW}

Thirty-six respondents (47\%) stated they have programs in place to reduce climbing-related injury and rescue. Four themes of climbing risk mitigation strategies emerged: coordination with climbing coalitions, permitting, preventative search and rescue (PSAR), and publication of accident reports.

\section{COORDINATION WITH CLIMBING COALITIONS}

Twenty-five percent (9 of 36) of responding areas' risk mitigation strategies involved coordination with climbing coalitions or alliances. Relationships with climbing coalitions varied, but commonalities included creation of climbing management plans and maintenance of effective communication with the climbing community. These climbing management plans frequently had bolt replacement and trail maintenance components. For instance, a county-managed climbing area in Iowa required their local climbing coalition to perform annual climbing infrastructure (eg, anchors) inspections and provide approval of new routes or infrastructure before route establishment.

Even when formal climbing management plans were not in place, climbing coalitions commonly served as a conduit between administrative agencies and climbing communities. Three respondents $(8 \%)$ explained that information on bolt and anchor replacement was collected through social media pages (Facebook and Mountain Project discussion boards) operated and monitored by climbing coalitions. Eleven respondents (30\%) described climbing area managers engaging in dialogue with climbing coalitions for notification about worn hardware, new hardware proposals, and hardware replacement. Survey respondents reported relying on climbing coalitions to communicate safety information to climbing visitors. Five respondents (14\%) stated that climbing coalitions were a "means of stay[ing] connected with 
Table 1. Presence of risk mitigation strategies assessed by demographic variables

\begin{tabular}{|c|c|c|c|c|}
\hline \multirow[t]{2}{*}{ Variable } & \multirow{2}{*}{$\frac{\text { All climbing areas }}{n=157}$} & \multicolumn{2}{|c|}{ Climbing areas with survey response } & \multirow[b]{2}{*}{$\mathrm{P}$-value } \\
\hline & & All areas $n=76$ & Areas with risk mitigation $n=36$ & \\
\hline Land area & & & & 0.2 \\
\hline Small & 48 & 25 & 14 & \\
\hline Medium & 58 & 25 & 12 & \\
\hline Large & 51 & 26 & 10 & \\
\hline Region & & & & 0.4 \\
\hline New England & 11 & 2 & 2 & \\
\hline Middle Atlantic & 4 & 1 & 0 & \\
\hline East North Central & 8 & 1 & 0 & \\
\hline West North Central & 17 & 6 & 4 & \\
\hline South Atlantic & 23 & 14 & 6 & \\
\hline East South Central & 7 & 3 & 1 & \\
\hline West South Central & 5 & 3 & 3 & \\
\hline Mountain & 58 & 40 & 17 & \\
\hline Pacific & 21 & 6 & 3 & \\
\hline \multicolumn{5}{|l|}{ Visitation } \\
\hline Low & - & $9^{a}$ & $3^{b}$ & 0.3 \\
\hline Medium & - & $16^{a}$ & $11^{b}$ & \\
\hline High & - & $9^{a}$ & $5^{b}$ & \\
\hline \multicolumn{5}{|l|}{ Composite risk index } \\
\hline Low & - & $13^{a}$ & $4^{b}$ & 0.4 \\
\hline Medium & - & $11^{a}$ & $7^{b}$ & \\
\hline High & - & $10^{a}$ & $8^{b}$ & \\
\hline
\end{tabular}

${ }^{a}$ The areas represented in these category totals are only those with a complete (100\% of questions) survey response $(\mathrm{n}=34)$.

${ }^{b}$ The areas represented in these category totals are only those with a complete (100\% of questions) survey response $(\mathrm{n}=19)$.

climbing communities" to ensure bidirectional communication. Two climbing coalitions were reported to run volunteer injury and rescue groups and provide education and training for climbers. Finally, 6 respondents $(20 \%)$ noted that local sports stores and local college- or university-led climbing groups were key resources for knowledge building and education around safe climbing.

\section{PERMITTING}

Eight respondents (22\%) included permitting systems as part of risk mitigation strategies. These respondents all reported that safety information on climbing risks and hazards was featured on climbing permits: part of their permit process required acknowledgement of risks and attestation prior to permit release. Certain areas, notably the Black Canyon of the Gunnison, require permits for all climbing. Two respondents noted that their areas only require permits for high-risk objectives. Two areas have separate sign-in processes at visitor centers, which includes signing liability waivers. This process enables them to create daily climber lists with locations to assist rangers. Three respondents $(8 \%)$ noted that permits are required for establishment of new routes-particularly if climbers intend to place new hardware.
Ten respondents (28\%) stated that commercial climbing supervised by outside guiding companies and organizations required permits. Two respondents $(6 \%)$ organized their own site-specific guide training courses. Official policies were reported across all entities managed by NPS and the USDAFS - with a few key exceptions - that required permits for guiding. It was also reported that federal policies govern the placement of fixed anchors or other forms of new hardware in designated wilderness areas managed by the NPS, USDAFS, and BLM. ${ }^{12}$ Prior authorization, provided by a park plan or case-by-case climber requests, is required for placement of new fixed anchors. ${ }^{12-14}$ For replacement of anchors in need of maintenance, authorization is only required in certain cases on a park-determined basis. ${ }^{15}$

\section{PREVENTATIVE SEARCH AND RESCUE}

Six respondents $(17 \%)$ cited PSAR as a risk mitigation strategy. Out of respondents who mentioned PSAR by name, $33 \%$ (2 of 6) referred to technical skill training and practice as PSAR, and 33\% cited conversations between rangers and climbers. The latter touched on a larger risk mitigation theme involving making ranger staff available for conversations. This came in the form of "Coffee with 
a Ranger" programming and ranger-led efforts to contact climbers before they head out to climb. Some examples include climbing rangers contacting climbers in the mornings before they head out for their climbs, advising climbers on route selection, providing information on route closures, and offering technical tips. Six respondents (20\%) explained how conversational-based PSAR can arise from more traditional patrol-based search and rescue models. Six respondents (20\%) emphasized the importance of the technical training local search and rescue organizations undergo as a means to bolster their climbing risk mitigation response.

\section{PUBLICATION OF ACCIDENT REPORTS}

Respondents also reported publicly publishing data on climbing-related injury and rescue as a method of risk mitigation. For example, respondents from El Dorado Canyon SP, Colorado, said they directly share accident data, new regulations, and safety concerns on educational signage in the park. Yosemite NP engages in a similar approach wherein they publish selected search and rescue incident reports on their website bimonthly during the peak season (roughly March to November). Thirty-four areas of study (22\%) were represented in accident reports submitted to the 2020 Accidents in North American Climbing by rescuers, rangers, and climbers. ${ }^{15}$

\section{LIMITATIONS TO CLIMBING RISK MITIGATION}

Ten respondents (13\%) endorsed having a fear of creating risk mitigation strategies, because this would inherently acknowledge a governing entity was condoning a risky sport, thus potentially exposing them to litigation. Three respondents $(4 \%)$ only agreed to complete the survey if guaranteed anonymity in their responses out of fear for litigation. Eight respondents (11\%) felt "torn" about the roles of the land management organizations - seeing their formal role as to protect resources, not individuals. Twenty-one respondents (28\%) lamented the absence of standard national systems for compiling and reporting climbing-specific injury and rescue data. Respondents stated that without formal reporting mechanisms, many injuries are unaccounted for, particularly when climbers self-rescue. Three respondents reported that climber reticence to disclose events resulting in injury might jeopardize future access to climbing routes.

\section{Discussion}

As rock climbing has become more popular, rates of climbing-related injury and rescue have increased..$^{1-3}$ Interventions capable of reducing the opportunity for injury and need for subsequent rescue are urgently needed. In surveying popular, publicly managed bouldering, traditional, and sport climbing areas, we found only $47 \%$ had programs in place to prevent climbing-related injury and rescue. These programs employed a range of risk mitigation strategies, with 4 themes: coordination with climbing coalitions $(25 \%)$, permitting (22\%), publication of accident reports $(22 \%)$, and PSAR (17\%). Identifying successful risk mitigation strategies, naming challenges, and sharing this information may help ensure useful risk mitigation techniques are adopted broadly.

Engagement with climbing coalitions was reported as advantageous by management entities. Coalitions provided an opportunity to outsource climbing-related risk mitigation efforts, while connecting and aligning management efforts with the needs of the local climbing community. For climbing areas that do not employ climbing-specific rangers, working with climbing coalitions may help ensure that climbing-related concerns and interests are communicated between climbers and land management organizations. These relationships may also provide opportunities for bidirectional information flow: Management entities can use coalitions to inform climbers of regulations and simultaneously receive information from climbers on successful or unsuccessful risk mitigation techniques. An additional benefit of partnering with climbing coalitions could be increased communication with other local entities, such as county search and rescue, which could be brokered through the climbing coalition.

Another commonly reported tactic was permitting. Through development of a permit system, management entities gain perspective on the frequency and volume of climbing use and a roster of climbers in an area. Such a technique may be particularly advantageous in areas where longer or more remote climbs are common, or conversely in areas or on routes with particularly high climber traffic. Having an in-person permit system also provides an opportunity for climber education. When picking up a permit, climbers may have the opportunity to talk with a ranger about their objective and gain information or insight that they might not have otherwise received. This process also serves to develop a personal connection between climbers and the personnel responsible for managing a given land area.

Likewise, the process of sharing accident or rescue data can help inform the climbing community about risks in a given area. However, it was clear from the survey respondents that there is no well-defined mechanism for public management entities to report these accidents, nor is there a central, publicly managed repository where these injuries can be compiled. Development of an anonymized, digital, curated injury repository may prove to 
be particularly useful. Building on the success of Accidents in North American Climbing, national climbing advocacy groups, public oversight agencies, or wilderness medicine organizations should be encouraged to consider such infrastructure to help guide assessments of climbing risk mitigation techniques.

\section{LIMITATIONS}

This research has several limitations. One limitation is in our modest response rate, which is subject to selection and nonresponse bias. There is also potential for recall bias given the survey design. Responses may have been biased due to the method of survey administration, with phone surveys biased toward socially desirable answers. Having individuals provide responses on behalf of land management organizations invited a proxy respondent effect. To encourage diverse responses reflecting strategies in the working context of specific areas, we allowed respondents to define risk mitigation programs on their own terms, which increased subjective variability. We were not able to quantify the amount that a given oversight entity depended on any given risk mitigation strategy, just its presence or absence. Finally, incidents in which climbers self-rescue were not captured in reported injury and rescue numbers.

\section{Conclusions}

Forty-seven percent of publicly managed climbing areas responding to our survey reported the presence of programs to prevent climbing-related injury and rescue. Existing programs involve coordination with climbing coalitions, permitting, preventative search and research, and publishing injury and rescue reports. There is considerable opportunity to evaluate the efficacy of risk mitigation strategies through intra-area and inter-area assessments. A centralized climbing injury database may prove useful when assessing the impact of risk mitigation techniques.

Author Contributions: Study design (JHM, JDF); data collection (JHM); manuscript writing and editing (JHM, JDF). Approval of final manuscript (JHM, JDF).

Financial/Material Support: None.

Disclosures: None.

\section{References}

1. Forrester JD, Tran K, Tennakoon L, Staudenmayer K. Climbing-related injury among adults in the United States: 5-year analysis of the National Emergency Department Sample. Wilderness Environ Med. 2018;29(4):425-30.
2. Grønhaug G, Saeterbakken A. No pain no gain: a survey of use of healthcare and reasons not to seek healthcare by Norwegian climbers with chronic injuries. BMJ Open Sport Exerc Med. 2019;5(1):e00513.

3. Grønhaug G. Self-reported chronic injuries in climbing: who gets injured when? BMJ Open Sport Exerc Med. 2018;4(1):e000406.

4. Buzzacott P, Schöffl I, Chimiak J, Schöffl V. Rock climbing injuries treated in US emergency departments, 2008-2016. Wilderness Environ Med. 2019;30(2):121-8.

5. United States Department of Agriculture, Forest Service. National visitor use monitoring survey results national summary report: Data collected FY 2015 through FY 2019. Available at: https://www.fs.usda.gov/sites/default/files/20 19-National-Visitor-Use-Monitoring-Summary-Report.pdf. Accessed July 18, 2021.

6. Ziesler, PS. 2020. Statistical abstract: 2019. Natural resource data series. NPS/NRSS/EQD/NRDS-2020/1272. National Park Service. Fort Collins, Colorado. Available at: https:// irma.nps.gov/DataStore/Reference/Profile/2272525. Accessed July 18, 2021.

7. US Department of the Interior, Bureau of Land Management. Public land statistics 2018. Available at: https://www. blm.gov/sites/blm.gov/files/PublicLandStatistics2018.pdf. Accessed July 18, 2021.

8. United States Department of the Interior, Forest Service. Land areas of the National Forest System. Available at: https://www. fs.fed.us/land/staff/lar-index.shtml; 2019. Accessed July 18, 2021.

9. National Park Service, Land Resources Division. Summary of acreage. 2019 calendar year report. Available at: https:// www.nps.gov/subjects/lwcf/acreagereports.htm. Accessed July 18, 2021.

10. GeoHack.Version 14 [Computer software]. Oslo, Norway. 2020. Available at: https://bitbucket.org/magnusmanske/ geohack/src/master/. Accessed July 18, 2021.

11. US Census Bureau. US Department of Commerce Economics and Statistics Administration. Census regions and divisions of the United States. Available at: https://www2.c ensus.gov/geo/pdfs/maps-data/maps/reference/us_regdiv.pdf. Accessed July 18, 2021.

12. Sammartino M. To bolt or not to bolt: a framework for common sense climbing regulation. Available at: https://www.ameri canbar.org/groups/environment_energy_resources/publi cations/plr/20200824-to-bolt-or-not-to-bolt/. Accessed July 18, 2021.

13. Access Fund and American Alpine Club. (2015). Policy on fixed anchors [Press release]. Available at: https:// d1w9vyym276tvm.cloudfront.net/assets/pdf/AF-AAC_Fixed AnchorPolicy_20150428.pdf. Accessed November 11, 2020.

14. Keith J. What the new NPS wilderness climbing policy means for climbers and bolting. Vertical Times. 2013;97:8-10.

15. MacDonald D, ed. Accidents in North American Climbing. Golden, CO: American Alpine Club; 2020. 